\title{
Threonine 286 of fatty acid desaturase 7 is essential for $\omega-3$ fatty acid desaturation in the green microalga Chlamydomonas reinhardtii
}

\section{Jong-Min Lim ${ }^{1+}$, Jayaraman Vikramathithan ${ }^{1+}$, Kwon Hwangbo ${ }^{1,2}$, Joon-Woo Ahn ${ }^{3}$, Youn-Il Park ${ }^{2}$, Dong-Woog Choi ${ }^{4}$ and Won-Joong Jeong ${ }^{*}$ *}

${ }^{1}$ Sustainable Bioresource Center, Korea Institute of Bioscience and Biotechnology, Daejeon, South Korea

${ }^{2}$ Department of Biological Science, Chungnam National University, Daejeon, South Korea

${ }^{3}$ Advanced Radiation Technology Institute - Korea Atomic Energy Research Institute, Jeonbuk, South Korea

${ }^{4}$ Department of Biology Education, Chonnam National University, South Korea

\section{Edited by:}

Takashi Osanai, RIKEN Center for Sustainable Resource Sciences, Japan

\section{Reviewed by:}

Ji-Joon Song, Korea Advanced Institute of Science and Technology, South Korea

Daniela Morales-Sanchez, University of Nebraska-Lincoln, USA

\section{${ }^{*}$ Correspondence:}

Won-Joong Jeong, Sustainable Bioresource Research Center, Korea Institute of Bioscience and Biotechnology, 111 Gwahangno, Yuseong-gu, Daejeon, South Korea e-mail:wonjoong@kribb.re.kr

${ }^{t}$ These authors have contributed equally to this work.
Omega-3 fatty acid desaturases catalyze the conversion of dienoic fatty acids (C18:2 and C16:2) into trienoic fatty acids (C18:3 and C16:3), accounting for more than $50 \%$ of the total fatty acids in higher plants and the green microalga Chlamydomonas reinhardtii. Here, we describe a Thr residue located in the fourth transmembrane domain of fatty acid desaturase 7 (FAD7) that is essential for the biosynthesis of $\omega-3$ fatty acids in $C$. reinhardtii. The $\omega-3$ fatty acid deficiency in strain CC-620, which contains a putative missense mutation at Thr286 of CrFAD7, was recovered by the overexpression of CC-125 CrFAD7. A Ser substitution in position 286 was able to partially complement the phenotype of the $\omega-3$ fatty acid deficiency, but other substitution variants, such as Tyr, His, Cys, and Gly, failed to do so. Prediction of the phosphorylation target site revealed that Thr286 may be phosphorylated. Analysis of the structural conformation of CC-620 CrFAD7 via topology prediction (and bends in the helix) shows that this missense mutation may collapse the catalytic structure of CrFAD7. Taken together, this study suggests that Thr286 is essential for the maintaining the catalytic structure of CrFAD7.

Keywords: CrFAD7, Thr286, fatty acid desaturase, topology, Chlamydomonas reinhardtii

\section{INTRODUCTION}

Fatty acids are the primary components of cell membranes. Longchain unsaturated fatty acids are important constituents of cellular membranes and play significant roles in maintaining membrane fluidity in many organisms. Polyunsaturated fatty acids, including $\alpha$-linolenic acid, are important for low-temperature responses (Gibson et al., 1994), sperm and pollen viability (Horiguchi et al., 1996; McConn and Browse, 1996; Wathes et al., 2007), and defense signaling (Kirsch et al., 1997; Wallis and Browse, 2002) in various organisms.

Membrane glycerolipid molecules comprise high levels of trienoic fatty acids, and more than $2 / 3$ of the fatty acids available in thylakoid membranes are C18:3 or a combination of C18:3 and C16:3 (Routaboul et al., 2012). The $\omega-3$ fatty acid desaturases (FADs) catalyze the conversion of dienoic fatty acids (C18:2 and C16:2) into trienoic fatty acids (C18:3 and C16:3). In higher plants, three $\omega-3$ FADs-FAD3, FAD7, and FAD8were identified and characterized (Wallis and Browse, 2002). FAD3 is specific for the endoplasmic reticulum, whereas FAD7 and its cold-inducible isozyme, FAD8, are plastid specific (Wallis and Browse, 2002). Although a large number of different FADs have been characterized from a variety of organisms, some of the more specialized enzymes are still being discovered. In the model microalga Chlamydomonas reinhardtii, $60 \%$ of total fatty acids are composed of $\omega-3$ and $\omega-6$ polyunsaturated fatty acids, of which more than $80 \%$ are $\omega-3$ species (Giroud and Eichenberger, 1988; Siaut et al., 2011). The CrFAD7 is the only $\omega-3$ FAD in $C$. reinhardtii and may play a similar role in low-temperature responses as in higher plants (Nguyen et al., 2013). Recently, it was reported that the CC-620 strain is a $\omega-3$ fatty acid-deficient mutant (Pflaster et al., 2014); the segregation analysis of CC- $620 \times$ CC-125 implicated that the deficiency is attributable to a missense mutation at Thr286 in CrFAD7.

Fatty acid desaturases are grouped into soluble and integral membrane classes in higher plants (Shanklin and Somerville, 1991). The soluble acyl-carrier-protein (ACP) desaturase enzymes are found in the plastids of higher plants, whereas the more extensive group of integral membrane acyl-CoA desaturases exist in endomembrane systems in both prokaryotes and eukaryotes (Shanklin and Cahoon, 1998). Most of the membranebound desaturases are homodimeric proteins that contain four membrane-spanning domains and three His box motifs [HX34H, HX2-3HH, and (H/Q)X2-3HH] (Shanklin, 1994). The di-iron active site of these enzymes is buried within a core four-helix bundle and positioned alongside a deep, bent, narrow hydrophobic cavity in which the substrate is bound during catalysis (Shanklin, 1994). Similarly, membrane receptors and channel proteins are integral membrane proteins composed of several transmembrane (TM) $\alpha$-helices that assemble through tertiary or quaternary 
structures to form bundles that cross the lipid bilayer ( $\mathrm{Pal}-$ czewski et al., 2000; Jiang et al., 2003). The biological function of these proteins involves conformational rearrangement of this TM bundle.

In higher plants and green algae, FAD7 also has four TM domains and His motifs. In addition, FAD7 has wellconserved Thr residues that are located distal to the active catalytic site; the roles of these Thr residues have not been studied (Shanklin, 1994; Pflaster etal., 2014). In the present study, we show that Thr286 is essential for the desaturation activity of CrFAD7 using complementation analyses with various modified FAD7s. We then discussed for the critical role of Thr286 in the maintenance of the active catalytic strucuure of CrFAD7 using prediction analysis of phosphorylation and topology. This study will help identify the physiological roles of $\omega-3$ fatty acids in microalgae and lead to an understanding of the structure and function of membrane-bound desaturases.

\section{MATERIALS AND METHODS}

\section{C. reinhardtii GROWTH CURVES AND CULTURE CONDITIONS}

Chlamydomonas reinhardtii cultures were maintained on Trisacetate-phosphate (TAP) agar medium at $25^{\circ} \mathrm{C}$ under continuous light. A single colony of $C$. reinhardtii was used to make a starter culture grown in liquid TAP medium. The starter culture was grown under 4 days of light on a rotary shaker $(200 \mathrm{rpm})$. Fresh TAP liquid medium $(50 \mathrm{ml})$ was prepared in $250 \mathrm{ml}$ Erlenmeyer flasks. This starter culture was inoculated at an $\mathrm{OD}_{750}$ of 0.05 . The growing cells were measured daily with an UV-visible spectrophotometer at $750 \mathrm{~nm}$.

\section{VECTOR CONSTRUCTION AND TRANSFORMATION}

The coding region of CrFAD7 (XM_001689611) cDNA was amplified by reverse transcription PCR (RT-PCR) using the primers 5'-CAT ATG CAG TGC CTG TCT CGC TCC A$3^{\prime}$ and $5^{\prime}$-GGGATATCTTAGGCCTTGCCGGCAA-3' (restriction sites underlined). CrFAD7 genomic DNA was amplified by PCR using the following primers: $5^{\prime}$-ACAT ATG CAG TGC CTG TCT CGC TCC A-3' and 5'-AGAT ATC GCC GTG CCA GAG TCT AAC T-3' (restriction sites underlined). The CrFAD7 cDNA and genomic DNA were cloned into expression vector pCr112 at the NdeI and EcoRV sites. This construct consists of the psaD promoter, CrFAD7, and the $p s a \mathrm{D}$ terminator along with the hygromycin resistance gene for selection, which is regulated by the $\beta$-tub promoter and the rbcS2 terminator. The construct was transformed into strain CC-620 using the glass bead method (Kindle, 1990). After transformation, cells were cultured in the dark without shaking for 2 days. The selection procedure was performed on agar medium containing $15 \mu \mathrm{g} / \mathrm{ml}$ hygromycin, and the plates were incubated under dim light for 2 weeks.

\section{RNA AND DNA ISOLATION}

Total RNA was prepared from $C$. reinhardtii cells $\left(\sim 1 \times 10^{7}\right.$ cells $)$ using the TRIzol reagent (Invitrogen, Carlsbad, CA, USA) according to the manufacturer's instructions. Five micrograms of total RNA were treated with 1 U RNase-free DNase (TaKaRa, Osaka, Japan) for $30 \mathrm{~min}$, and purification was performed according to the manufacturer's instructions. For the isolation of genomic DNA, $500 \mu \mathrm{l}$ TEN buffer (10 mM EDTA, $10 \mathrm{mM}$ Tris-HCl, and $150 \mathrm{mM} \mathrm{NaCl})$ was added to samples $\left(\sim 1 \times 10^{7}\right.$ cells $)$. Cells were harvested and resuspended in $150 \mu \mathrm{l} \mathrm{H}_{2} \mathrm{O}$ and $300 \mu \mathrm{l}$ SDS-EB buffer (2\% SDS, $400 \mathrm{mM} \mathrm{NaCl}, 100 \mathrm{mM}$ Tris-HCl, $\mathrm{pH}$ 8.0, and $40 \mathrm{mM}$ EDTA). Extraction was carried out first with $350 \mu \mathrm{l}$ phenol:chloroform:isopropanol $(24: 24: 1, \mathrm{v} / \mathrm{v} / \mathrm{v})$ and then with $300 \mu \mathrm{l}$ chloroform:isopropanol (24:1, v/v). Genomic DNA was precipitated with two volumes of absolute ethanol and washed with $70 \%$ ethanol. The DNA pellet was resuspended in Tris-EDTA buffer.

\section{RT-PCR ANALYSES}

Reverse transcription was carried out using $2 \mu \mathrm{g}$ total RNA, $50 \mu \mathrm{M}$ oligo (dT), $200 \mathrm{U}$ M-MLV reverse transcriptase (Promega, Madison, WI, USA), $500 \mu \mathrm{M}$ each dNTP, and $20 \mathrm{U}$ ribonuclease inhibitor. For semi-quantitative RT-PCR, cDNA was amplified with $1.5 \mathrm{U}$ Ex Taq DNA polymerase (TaKa Ra), $100 \mu \mathrm{M}$ each $\mathrm{dNTP}$, and 10 pmol each gene-specific primer using a T1 thermal cycler (Biometra GmbH, Gottingen, Germany). Amplification was performed with $25-35$ cycles of $94^{\circ} \mathrm{C}$ for $30 \mathrm{~s}, 56^{\circ} \mathrm{C}$ for $30 \mathrm{~s}$, and $72^{\circ} \mathrm{C}$ for $30 \mathrm{~s}$. To detect transcripts, the following primers were used: CrFAD7 forward primer: 5' ${ }^{\prime}$ TTCACCGCTGAGCGCAA-3'; reverse primer: 5'-GTGGGTGCCGATGTCGTGGT-3'; CrFAD2 forward primer: $5^{\prime}$-CTTCACCAAGCGCGAGCGCA-3'; reverse primer: $5^{\prime}$-CTTGATGGCCTCGGTGGCCT-3'; Cr $\triangle 4 F A D$ forward primer: $5^{\prime}$-CACCTTCGCCGTGTCGCACA-3'; reverse primer: 5'-CATCTCGCCGTCGCGCTTGA-3'; CrFAD6 forward primer: 5'-ATGGCCAAGTGGGACTCCAC-3'; reverse primer: 5'-CCACGGTGAAGGTGCTCATC-3'; CrFAD13 forward primer: 5'-CATGATCTCGCCCCTTAGCTACTT-3'; reverse primer: $5^{\prime}$-GTCCATCTGAATGTGGGACACCT- $3^{\prime}$; $\alpha$-tubulin forward primer: 5'-CTCGCTTCGCTTTGACGGTG-3'; reverse primer: 5'-AACGTCCTTGGGCACGACGT -3'; and IAD5 forward primer: 5'-GCGAGGTCTCTGCTCTGGTG-3'; reverse primer: 5' -TACTCGGACTTGGCGATCCA -3' . To detect CrFAD7 cDNA transcripts in transgenic lines, specific primers [for-

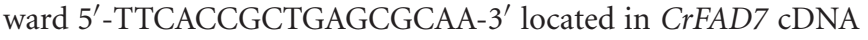
and reverse $5^{\prime}$-CCTGTGGCTAATTGACCGTG-3' located in psaD terminator] were used.

\section{ANALYSIS OF FATTY ACID METHYL ESTERS BY GAS CHROMATOGRAPHY}

Total lipids were extracted from $20 \mathrm{mg}$ freeze-dried samples according to a previously described extraction method (Sasser, 1990). Saponification was performed with $2 \mathrm{ml}$ saponification reagent (7.5 M NaOH: $\left.\mathrm{CH}_{3} \mathrm{OH}, 1: 1, \mathrm{v} / \mathrm{v}\right)$ at $100^{\circ} \mathrm{C}$ for $30 \mathrm{~min}$. For the production of fatty acid methyl esters, $4 \mathrm{ml}$ methylation reagent $\left(\mathrm{CH}_{3} \mathrm{OH}: 6 \mathrm{~N} \mathrm{HCl}, 1: 1, \mathrm{v} / \mathrm{v}\right)$ were added to the saponified sample and incubated at $80^{\circ} \mathrm{C}$ for $10 \mathrm{~min}$. After the reaction, $2.5 \mathrm{ml}$ extraction solvent hexane:methyl tetra-butyl ether, $1: 1$, $\mathrm{v} / \mathrm{v}$ ) were added and incubated with shaking for $10 \mathrm{~min}$. The upper phase was separated by centrifugation at 4,000 rpm for $10 \mathrm{~min}$. A washing step was carried out with $6 \mathrm{ml}$ washing solution $(0.5 \mathrm{M} \mathrm{NaOH})$. The fatty acid methyl esters were analyzed by gas chromatography (model YL-6100GC; Young Lin Science, Anyang, Korea) equipped with a flame ionization detector and an 

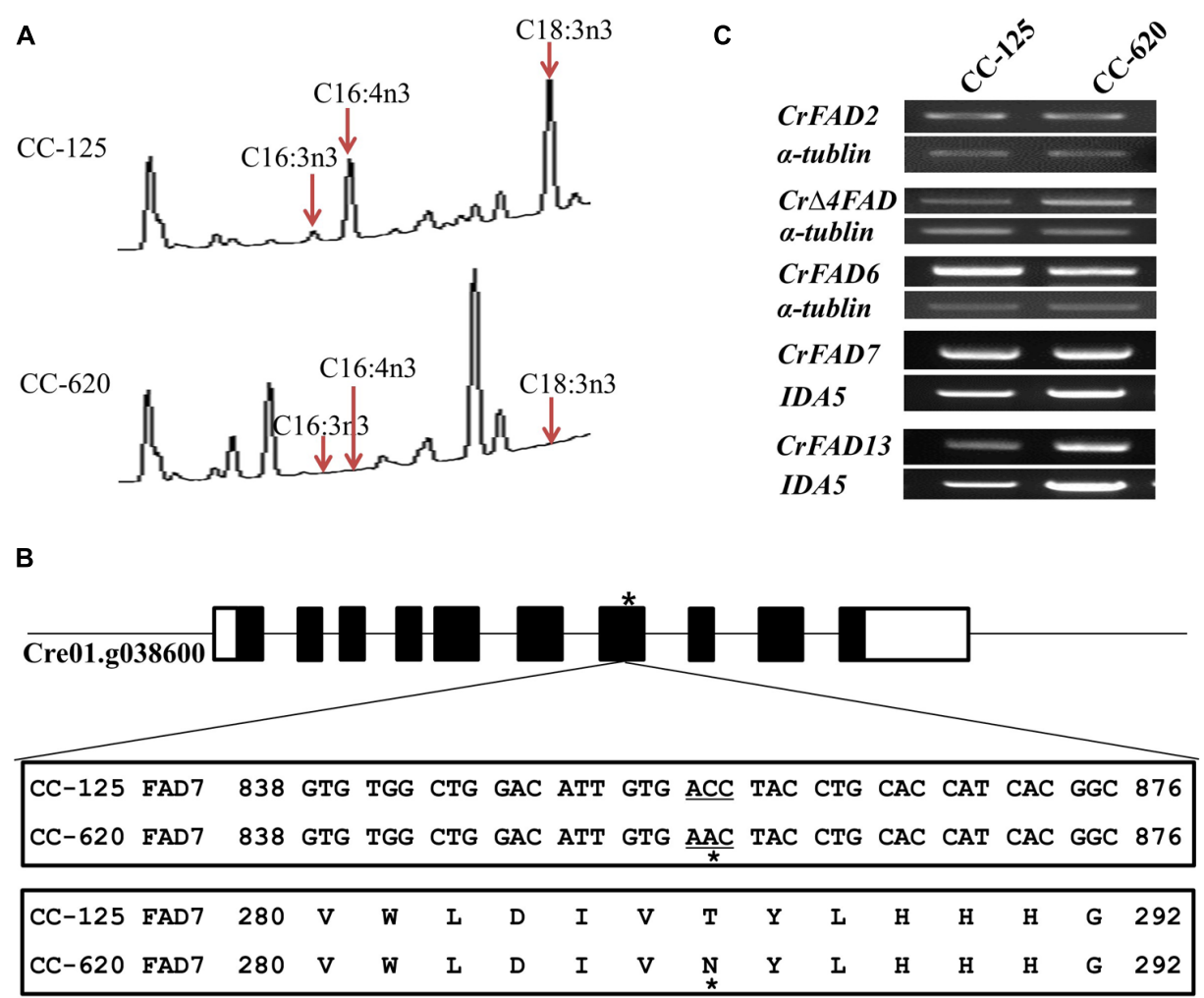

FIGURE 1 | (A) Comparison of fatty acids profiles in strains CC-125 and CC-620 from gas chromatographic analyses. Arrows indicate significant changes in specific fatty acids. (B) Sequence comparison (nucleotide and amino acid) of CrFAD7 in CC-620 and other wild-type strains (CC-124, CC-125, and CC-621). The black square boxes indicated the exon number or gene locus of the CrFAD7 and star indicates the missense mutation (Thr286Asn) caused by a point mutation. (C) RT-PCR analyses of fatty acid desaturases (FADs). CrFAD2 (EU596472), CrFAD7 (XM-001689611), CrFAD6 (AB007640), CrFAD13 (AB239770), and $\triangle-4 F A D$ (JN089704). Genes for IAD5 and $\alpha$-tubulin served as internal controls.
INNOWAX capillary column (Agilent Technologies, Santa Clara, CA, USA; $30 \mathrm{~m} \times 0.32 \mathrm{~mm} \times 0.5 \mu \mathrm{m})$. Each fatty acid methyl ester component was identified and quantified using the Supelco ${ }^{\circledR} 37$ Component Fatty Acid Methyl Ester Mix (Sigma).

\section{SOUTHERN AND NORTHERN ANALYSES}

Ten micrograms of genomic DNA were digested with $K p n \mathrm{I}$ and separated by $0.8 \%$ agarose gel electrophoresis. The separated DNA was blotted onto a Hybond $\mathrm{N}^{+}$nylon membrane (Amersham Biosciences, Piscataway, NJ, USA). A 0.3-kb PCR fragment corresponding to the $C$-terminal region of $C r F A D 7$ was used as a probe. ${ }^{32} \mathrm{P}$-labeled probes were produced using the Rediprime ${ }^{\mathrm{TM}}$ II Random Labeling System (Amersham Biosciences), and hybridization was performed according to the manufacturer's instructions. Signals were detected using the Bio-Imaging Analyzer BAS-1800II (Fuji, Tokyo, Japan).

Total RNA was extracted from $C$. reinhardtii cells ( $5 \mathrm{ml}$ liquid culture) using the TRIzol reagent (Invitrogen, Carlsbad, CA, USA) according to the manufacturer's instructions. Twenty micrograms of total RNA were separated by gel electrophoresis and blotted onto a Hybond-N nylon membrane (Amersham Biosciences, USA) by capillary transfer. A $0.3-\mathrm{kb}$ fragment corresponding to the CrFAD7 cDNA was used as a probe. Procedures for hybridization and signal detection were as described above.

\section{IN SILICO ANALYSES}

To identity conserved Thr residues of $C$. reinhardtii FAD7, we used CrFAD7 as bait and employed the BLASTP program (BLASTP 2.2.26+) hosted at Phytozome, version 9.1 (http://www.phytozome.net). Clustal W2.1 was used for multiple sequence alignment. The accession numbers of the FAD7-homologous genes used in this study were as follows: VcFAD7 (XP_002953984), CnFAD7 (EF1N50714), CosFAD7 (EIE21058), CrFAD7 (XP_001689663), OsFAD7 (NP_001060733), PpFAD7 (XP_001752878), AtFAD7 (NP187727), GmFAD7 (NP-001237838), ZmFAD7 (NP-001105303), and SspDesA (CAA37584). Prediction analyses for phosphorylation target sites and topology were performed using the online software tools Kinase phos2 and TMHMM (Version 1), respectively. Default settings of both programs were used.

\section{RESULTS}

\section{THE $\omega-3$ FATTY ACID DEFICIENCY IN THE CC-620 STRAIN IS COMPLEMENTED BY CC-125 CrFAD7 GENE EXPRESSION}

We confirmed the $\omega-3$ fatty acid deficiency in the CC-620 strain obtained from the Chlamydomonas Resource Center (Figure 1A). The point mutation in the seventh exon of the CrFAD7 gene, which causes a missense mutation (Thr286Asn), was confirmed to be identical to that described in the study by 

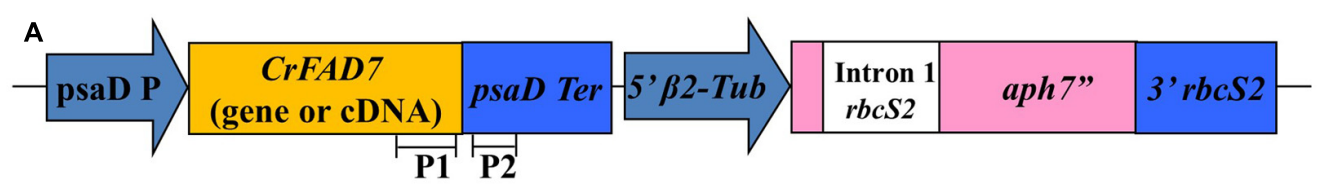

B

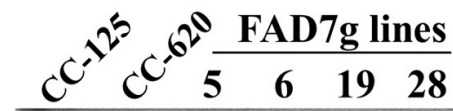

D

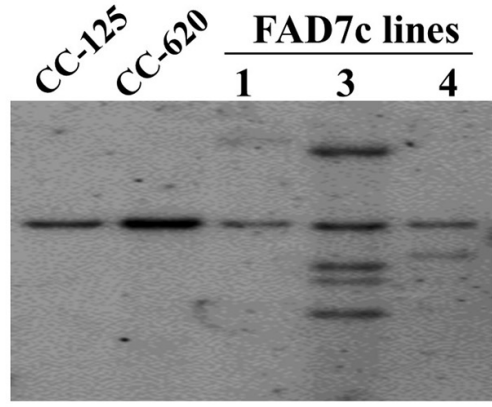

FIGURE 2 | Generation of CC-125 CrFAD7-expressing transgenic lines using CC-620. (A) Schematic diagram of the transformation vector. P1 and $\mathrm{P} 2$ indicate the regions used as probes for Southern or northern blot analysis. (B,D) Southern blot analysis of the CrFAD7 gene. Genomic DNA was digested by Xbal and blotted against probe P1. (C) Northern blot analysis to identify the expression of CrFAD7 mRNA in the CrFAD7 genomic
(Pflaster etal., 2014; Figure 1B). To identify the main cause for abnormal $\omega-3$ fatty acid desaturation, transcript levels were investigated for CrFAD2 (EU596472), Cr $\triangle F A D 4$ (JN089704), CrFAD6 (AB007640), CrFAD7 (XM_001689611), and CrFAD13 (AB239770), whose expression products are either directly or indirectly involved in $\omega-3$ fatty acid biosynthesis. However, RT-PCR analysis detected no significant differences in the expression levels of these genes between the CC-620 and CC-125 strains (Figure 1C). Next, we sequenced the cDNAs of the above-mentioned genes. Except for the point mutation in the CrFAD7 gene, no mutations were observed in the CrFAD2, Cr $\triangle F A D 4$, CrFAD6, or CrFAD13 genes.

To reveal the phenotype of the $\omega-3$ fatty acid deficiency in strain CC- 620 by the missense mutation detected in CrFAD7, genetic transformation of CC-620 was carried out using genomic DNA and cDNA of CC-125 CrFAD7. Transgene insertion and expression were confirmed by Southern, Northern, and RT-PCR analyses (Figures 2A-E).

In CrFAD7 genomic DNA-complemented strains (in transgenic lines), the normal fatty acid composition was recovered
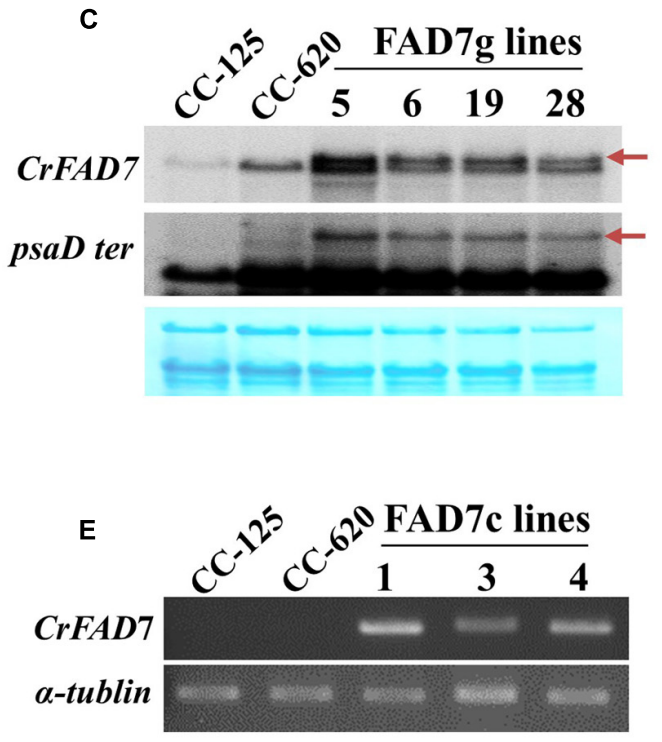

DNA-expressing transgenic lines; P1 probe for CrFAD7 transcript or P2 probe for $3^{\prime}$ UTR (PsaD terminator) of CrFAD7 transcript. Arrows indicate transcripts of transformed CrFAD7 gene. (E) RT-PCR analysis to identify transcripts of transformed CrFAD7 cDNA. $\alpha$-tubulin served as the internal control. FAD7g lines: complementation lines with FAD7genomic DNA; FAD7c lines: complementation lines with FAD7 cDNA.

with various levels of $\mathrm{C} 16: 4$ and $\mathrm{C} 18: 3 \mathrm{n} 3$; in comparison with CC- 125 , more than $80 \%$ of $\omega-3$ fatty acids were recovered with a concomitant reduction of $\omega-6$ fatty acids in several transgenic lines (Figures 3A,B). The $\omega-3$ fatty acids hexadecatrienoic acid (C16:3n3), hexadecatetraenoic acid (C16:4), and $\alpha$-linolenic acid (C18:3n3) were not detected in the CC-620 strain, whereas, the $\omega-3$ fatty acids were found to be $1.9,12.7$, and $20.5 \mathrm{~mol} \%$ in CC125 , and to be $1.1,9.3$, and $17.7 \mathrm{~mol} \%$ in the complemented strain (Figure 3; Table 1). The concomitant reduction in $\omega-6$ fatty acids was detected in the complemented transgenic lines compared to the CC-620 strain, but no significant differences in either saturated $(\mathrm{C} 16: 0)$ or monounsaturated fatty acid accumulation were observed in CC-125, CC-620, or the transgenic lines. In CrFAD7 cDNA-complemented strains, the recovery level of $\omega-3$ fatty acids was lower than that of CrFAD7 genomic DNA-complemented lines (Figures 3A,B).

\section{Thr286 IS CONSERVED IN HIGHER PLANTS AND GREEN ALGAE}

Fatty acid desaturase 7 is a membrane-bound desaturase, but its crystal structure has not yet been solved. Analysis of conserved 


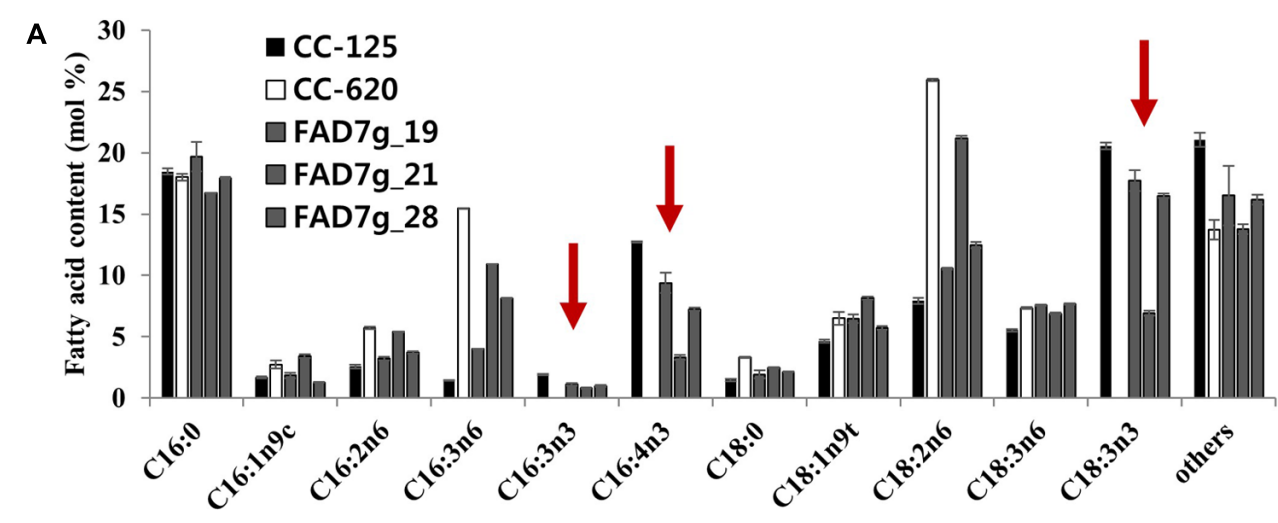

B

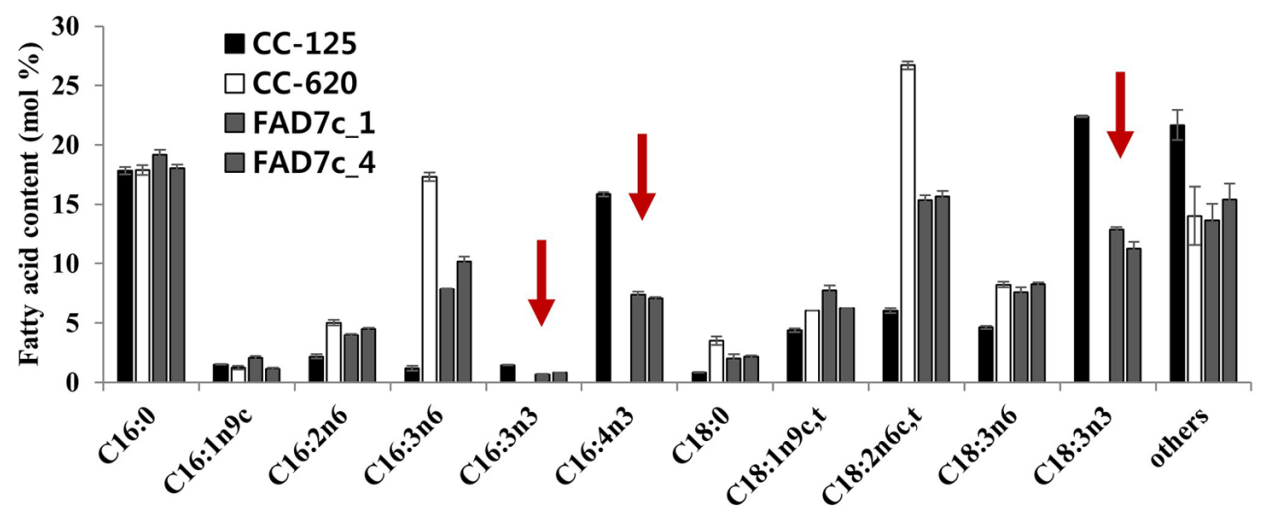

FIGURE 3 | Complementation of $\omega 3$-fatty acid deficiency in strain CC-620 by expression of the CC-125 CrFAD7 gene. Fatty acid compositions in CC-125, CC-620, and complementary strains were compared.

(A) Complementation using CrFAD7 genomic DNA. FADg_19, FADg_21, and
FADg_28 complementation strains expressing CrFAD7 genomic DNA (B) Complementation using CrFAD7 cDNA. FAD7c_1 and FADc_4 complementation strains expressing CrFAD7 cDNA. The values represent the mean \pm standard error (SEM). The experiments were conducted in triplicate. sequence motifs in FAD7 shows that the four membrane-spanning domains and three His box motifs are conserved in all FAD7 from microalgae to higher plants (Figure S1). Interestingly, the Thr residue in the fourth TM domain is specifically well conserved (Figure S1). The membrane protein topology of CrFAD7 was predicted using TMHMM. Topology prediction of CrFAD7 detected clear differences between CC-125 and CC-620 CrFAD7 (Figures S2A,B). A substitution of Ser286 for Thr286 showed a similar pattern as CC-125 CrFAD7 (Figures S2A,C). In CC-125 and Ser286-substituted CrFAD7, all four TM helices were linearly arranged, and the $C$-terminal region containing the third His box motif after the fourth TM domain was predicted to be located inside the membrane (Figures S2A,C). However, in CC$620 \mathrm{CrFAD} 7$, the $C$-terminal region was predicted to be located outside of the membrane (Figure S2B), indicating that the fourth TM domain changes (or loses) its structural helix.

To test the above prediction that Thr could be replaced by Ser, we transformed the CC-620 strain with modified CrFAD7 genes that would encode Ser, Tyr, His, Cys, or Gly substitutions at position 286. Only the Ser-substituted transgenic lines recovered the missing fatty acids (Figure 4) but other substitution variants, such as Tyr, His, Cys, and Gly, failed to do so (Figure S3). However, the accumulation of fatty acids was partially complemented compared with CC-125. The accumulation of $\alpha$-linolenic acid approached $3 \%$ in the Ser-substituted transgenic lines, whereas the accumulation approached $24 \%$ in strain CC-125. These results suggest that Thr286 is critical for proper CrFAD7 activity. To address the possible phosphorylation of Thr286 in silico, amino acid sequences of CC-620 and CC-125 CrFAD7 were analyzed using a phosphorylation site prediction tool, Kinase phos2. The result predicted 21 sites of phosphorylation, including Thr286, in CrFAD7 targeted by protein kinase $\mathrm{B}$ or $\mathrm{G}$ protein-coupled receptor kinase (Figure S4A). Only 20 target sites were predicted in CrFAD7 of strain CC620 because of the absence of Thr286 in the CrFAD7 CC-620 strain (Figure S4B). Ser286 was predicted as a phosphorylation target for AKT1, a type of protein kinase B, but not for $\mathrm{G}$ protein-coupled receptor kinase (data not shown). In addition, the conserved Thr residues of all FAD7s (mentioned in Figure S1) were predicted to be phosphorylation targets (data not shown).

\section{DISCUSSION}

In this study, our complementation results with the modified FAD7s indicated that Thr286 is essential for CrFAD7 desaturation activity (Figures 3 and 4). Topology and phosphorylation prediction showed that the missense mutation may disrupt the catalytic structure of the $\omega-3$ FAD (Figures S2 and S4). 
Table 1 | Fatty acid composition of CC-125, CC-620 and one of complemented strains analyzed through GC-FID.

\begin{tabular}{llll}
\hline Fatty acid composition & CC-125 & CC-620 & FAD7g_19 \\
\hline C16:0 & $18.48 \pm 0.251$ & $18.01 \pm 0.292$ & $19.68 \pm 1.188$ \\
C16:1n9c & $1.67 \pm 0.074$ & $2.73 \pm 0.336$ & $1.86 \pm 0.22$ \\
C16:2n6 & $2.57 \pm 0.13$ & $5.7 \pm 0.098$ & $3.21 \pm 0.148$ \\
C16:3n6 & $1.44 \pm 0.037$ & $15.47 \pm 0.018$ & $4 \pm 0.036$ \\
C16:3n3 & $1.93 \pm 0.058$ & ND & $1.11 \pm 0.093$ \\
C16:4n3 & $12.73 \pm 0.067$ & ND & $9.38 \pm 0.83$ \\
C18:0 & $1.47 \pm 0.093$ & $3.32 \pm 0.061$ & $1.93 \pm 0.327$ \\
C18:1n9t & $4.63 \pm 0.129$ & $6.5 \pm 0.533$ & $6.46 \pm 0.357$ \\
C18:2n6 & $7.93 \pm 0.246$ & $25.95 \pm 0.089$ & $10.56 \pm 0.083$ \\
C18:3n6 & $5.52 \pm 0.104$ & $7.33 \pm 0.083$ & $7.57 \pm 0.039$ \\
C18:3n3 & $20.56 \pm 0.296$ & ND & $17.74 \pm 0.852$ \\
others & $21.07 \pm 0.574$ & $13.73 \pm 0.794$ & $16.51 \pm 2.434$ \\
FAME & $100 \pm 2.059$ & $98.74 \pm 2.555$ & $100.01 \pm 6.607$ \\
\end{tabular}

ND, not detected.

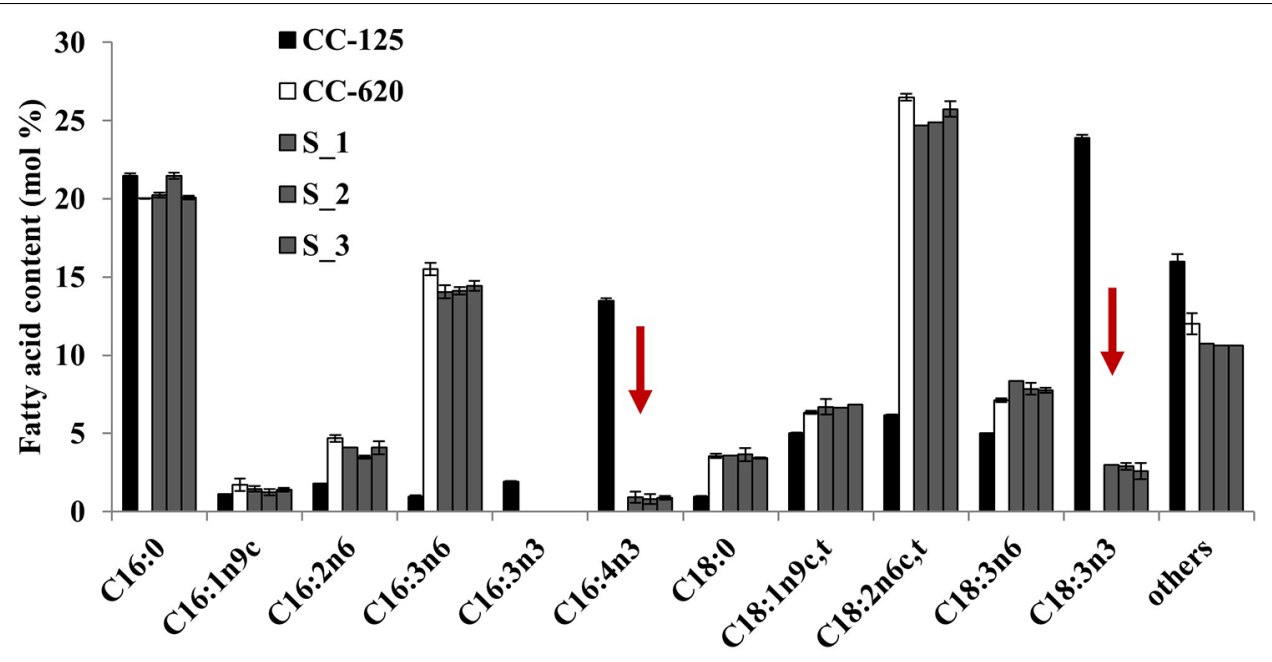

FIGURE 4 | Fatty acid composition of strain CC-620 and serine substituted lines (S_1, S_2, and S_3) expressing CrFAD7 cDNA. The values represent the mean \pm standard error (SEM). The experiments were conducted in triplicate.

Chlamydomonas reinhardtii strain CC-620 is a high-efficiency mating strain that produces autolysin and has been used previously as a common laboratory wild-type strain. Recently, Pflaster et al. (2014) reported that the $\omega-3$ fatty acid deficiency in $C$. reinhartii strain CC-620 correlated with a single locus, possibly attributed the missense mutation (Thr286Asn) in CrFAD7. In the present study, our complementation results provide direct evidence that the missense mutation is the real cause of the $\omega$ 3 fatty acid deficiency in CC-620 (Figures 3 and 4). $\omega-3$ fatty acids deficiency of CC-620 can be recovered by nuclear transformation of a wild type (CC-125) CrFAD7 gene. Interestingly, when a cDNA encoding CC-125 CrFAD7 was used for complementation, the transformed CC-620 produced $60 \% \omega-3$ fatty acids compared to strain CC-125, and transcription could be detected by RT-PCR. However, when genomic DNA encoding CrFAD7 was used, several transformed strains produced nearly maximal levels of $\omega-3$ fatty acids, and transcription was clearly detected by Northern blot analyses (Figure 2C). These results suggest that a genomic fragment corresponding to CrFAD7 efficiently expresses the CrFAD7 transcript compared to cDNA, and that the transcript level of CrFAD7 is proportional to $\mathrm{FAD7}^{\prime} \mathrm{s}$ activity.

Impaired progeny production was observed when strain CC620 was mated with its opposite mating type (Goodenough et al., 2007). Interestingly, the involvement of $\omega-3$ fatty acids in reproduction was also reported in higher plants and animals. The Arabidopsis triple mutant for FAD3, 6, and 7 manifested as male sterility (McConn and Browse, 1996). The animal $\omega-3$ fatty acid 
deficiency resulted in impaired steroid hormone synthesis (Wathes et al., 2007). These findings suggest that $\omega-3$ fatty acids may be important for reproduction in microalgae, including C. reinhardtii. The strain CC-620 had the additional phenotypes of slow growth and increased sensitivity at high temperature $\left(37^{\circ} \mathrm{C}\right) \mathrm{com}-$ pared to the CC-125 strain (Figure S5). However, these phenotypes were recovered in the progenies of CC-620 × CC-124, which are missing $\omega-3$ fatty acids, indicating that the CC-620 genome contains another mutation(s) responsible for these phenotypes in addition to the FAD7 mutation.

Protein phosphorylation plays a role in various physiological responses by altering enzymatic activity, from active to inactive or vice versa (Hunter, 1995). The in silico prediction detected 21 and 20 Thr residues of CC-125 and CC-620 CrFAD7, respectively, as target phosphorylation sites for protein kinase $\mathrm{B}$ or G protein-coupled receptor kinase (Figure S4). Ser286 was predicted as a phosphorylation site. However, the replacement of Thr286 by Ser286 only partially recovered the activity of FAD7 in four transgenic lines (Figures 4 and S3). These results suggest that Thr and Ser at position 286 can be phosphorylated by kinases and that Thr is preferred and necessary for full activity of the FAD. A significant preference for Thr or Ser as the phosphoacceptor residue has been previously reported, but this selectivity and its mechanism is unknown (Marin et al., 1986; Kuenzel etal., 1987; Litchfield etal., 1990; Kõivomägi et al., 2013).

\section{POSSIBLE MECHANISMS OF CrFAD7 IN $\omega-3$ FATTY ACID SYNTHESIS}

In higher plants and microalgae, lipid-linked acyl desaturation is a well-known mechanism; this process is induced by FADs. All FADs have TM domains, His motif boxes, and a di-iron active site, which all play important roles in the desaturation process. Our results indicate that the conserved Thr286 in the fourth TM domain is essential for fatty acid desaturation. Thr is less polar than Ser, but both have similar structures (both contain a polar hydroxyl group), differing only in the presence of a methyl group attached to the $\beta$-carbon in Thr. A mutation of a polar amino acid in a TM helix has been reported to abolish its structure; Thr residues function cooperatively to maintain the normal structure of the TM domain through hydrogen bonding (Dawson et al., 2002). In our transgenic lines, due to the lack of this methyl group in Ser, Ser286 may not efficiently donate hydrogen molecules to maintain the correct structure of the TM domain and fails to recover the full activity of FAD7. The Thr286Asn missense mutation of the CrFAD7 protein may disrupt the structure of the TM domain, leading to a loss of CrFAD7 desaturation activity.

Mutation of Thr may directly affect the structure of the TM domain, as this single residue was able to stimulate significant helix associations in model peptides (Gratkowski et al., 2001). In the TM domain of $\beta 2$-adrenergic receptors, the statistical $g 2$ conformations of both Ser and Thr residues decreased their $f$ angles and increased their c angles; a hydrogen bond was formed between the $\mathrm{O} \gamma$ atoms of Ser and Thr, with the i-3 or i-4 carbonyl oxygen, inducing or stabilizing the angle in the helix $3-4^{\circ}$ larger than for Ala (Ballesteros et al., 2000). This finding indicates that the local alterations in Ser and Thr in the TM domain may result in significant conformational changes across TM helices.
Based on our results, the loss of function in CC-620 CrFAD7 could be explained by a number of mechanisms. In CC-620 CrFAD7, the Thr286Asn mutation in the fourth TM domain may induce alteration of angle in the $\alpha$-helix. This altered bending would induce a change in CrFAD7's structural orientation. Structural shifts could deform the cooperative catalytic structure or cause the third $C$-terminal his motif box to be positioned too closed or too far from the catalytic active site. Alternately, the mutation may abolish the helix completely. Membrane topology prediction of the CC-620 CrFAD7 using TMHMM failed to detect a fourth helix. This would cause the third $C$-terminal his box to be located outside of membrane rather than inside (Figure S2B). In either scenario, the structural loss or alteration of the fourth TM domain would collapse the cooperative catalytic structure needed for $\omega-3$ fatty acid desaturation. In addition, the presence or absence of position 286 phosphorylation could also induce a structural change in the fourth TM domain and alter the topology. CrFAD7 Thr286 seems to be positioned away from the catalytic site, indicating that the phosphorylation may cause a structural change in the fourth TM domain rather than directly regulating enzymatic activity. To support this, previous studies have shown that phosphorylation by kinase can alter the protein conformation and modulate its biological function (Birck et al., 1999; Groban et al., 2006; Grosely et al., 2013). Any of these three possible mechanisms would lead to TM domain structural modification and prevent CC-620 CrFAD7 from synthesizing $\omega-3$ fatty acids.

In summary, we show that the CrFAD7 point mutation directly causes an alteration in the fatty acid profile of the CC-620 strain using complementation analysis with a CC-125 copy of CrFAD7. Amino acid substitutions and predictions of phosphorylation sites revealed that Thr286 may be a target of phosphorylation by protein kinase B or G protein-coupled receptor kinase. Both TM topology prediction and the structure of the TM $\alpha$-helix show that Thr286 may be essential for maintaining the correct catalytic structure of CrFAD7.

\section{ACKNOWLEDGMENTS}

This work was supported by a Grant from the Advanced Biomass R\&D Center (ABC) of Korea Grant funded by the Ministry of Education, Science and Technology (ABC-2011-0031343), a Grant from the Golden Seed Project, Ministry of Agriculture, Food, and Rural Affairs (MAFRA), the Ministry of Oceans and Fisheries (MOF), Rural Development Administration (RDA), and Korea Forest Service (KFS) and a Grant from the research program of Korea Atomic Energy Research Institute (KAERI), Republic of Korea.

\section{SUPPLEMENTARY MATERIAL}

The Supplementary Material for this article can be found online at: http://www.frontiersin.org/journal/10.3389/fmicb.2015.00066/ abstract

\section{REFERENCES}

Ballesteros, J. A., Deupi, X., Olivella, M., Haaksma, E. E. J., and Pardo, L. (2000). Serine and threonine residues bend $\alpha$-helices in the $\chi 1=\mathrm{g}$ - conformation. Biophys. J. 79, 2754-2760. doi: 10.1016/S0006-3495(00)76514-3 
Birck, C., Mourey, L., Gouet, P., Fabry, B., Schumacher, J., Rousseau, P., et al. (1999). Conformational changes induced by phosphorylation of the FixJ receiver domain. Structure 7, 1505-1515. doi: 10.1016/S0969-2126(00)88341-0

Dawson, J. P., Weinger, J. S., and Engelman, D. M. (2002). Motifs of serine and threonine can drive association of transmembrane helices. J. Mol. Biol. 316, 799-805. doi: 10.1006/jmbi.2001.5353

Gibson, S., Arondel, V., Iba, K., and Somerville, C. (1994). Cloning of a temperatureregulated gene encoding a chloroplast $\omega-3$ desaturase from Arabidopsis thaliana 1. Plant Physiol. 106, 1615-1621. doi: 10.1104/pp.106.4.1615

Giroud, C., and Eichenberger, W. (1988). Fatty acids of Chlamydomonas reinhardtii: structure, positional distribution and biosynthesis. Biol. Chem. Hoppe Seyler. 369, 18-19.

Goodenough, U., Lin, H., and Lee, J. (2007). Sex determination in Chlamydomonas. Semin. Cell Dev. biol. 18, 350-361. doi: 10.1016/j.semcdb.2007. 02.006

Gratkowski, H., Lear, J. D., and DeGrado, W. F. (2001). Polar side chains drive the association of model transmembrane peptides. Proc. Natl. Acad. Sci. U.S.A. 98 880-885. doi: 10.1073/pnas.98.3.880

Groban, E. S., Narayanan, A., and Jacobson, M. P. (2006). Conformational changes in protein loops and helices induced by post-translational phosphorylation. PLoS. Comp. Biol. 2:e32. doi: 10.1371/journal.pcbi.0020032

Grosely, R., Kopanic, J. L., Nabors, S., Kieken, F., Spagnol, G., Al-Mugotir, M., et al. (2013). Effects of phosphorylation on the structure and backbone dynamics of the intrinsically disordered connexin43 C-terminal domain. J. Biol. Chem. 288, 24857-24870. doi: 10.1074/jbc.M113.454389

Horiguchi, G., Kodama, H., Nishimura, M., and Iba, K. (1996). Role of $\omega-3$ fatty acid desaturases in the regulation of the level of trienoic fatty acids during leaf cell maturation. Planta 199, 439-442. doi: 10.1007/BF00195737

Hunter, T. (1995). Protein kinases and phosphatases: the yin and yang of protein phosphorylation and signaling. Cell 80, 225-236. doi: 10.1016/00928674(95)90405-0

Jiang, Y., Lee, A., Chen, J., Ruta, V., Cadene, M., Chait, B. T., et al. (2003). X-ray structure of a voltage-dependent K+ channel. Nature 423, 33-41. doi: 10.1038 /nature 01580

Kindle, K. L. (1990). High-frequency nuclear transformation of Chlamydomonas reinhardtii. Proc. Natl. Acad. Sci. U.S.A. 87, 1228-1232. doi: 10.1073/pnas.87.3.1228

Kirsch, C., Takamiya-Wik, M., Reinold, S., Hahlbrock, K., and Somssich, I. E. (1997). Rapid, transient, and highly localized induction of plastidial $\omega-3$ fatty acid desaturase mRNA at fungal infection sites in Petroselinum crispum. Proc. Natl. Acad. Sci. U.S.A. 94, 2079-2084. doi: 10.1073/pnas.94.5.2079

Kõivomägi, M., Örd, M., Iofik, A., Valk, E., Venta, R., Faustova, I., et al., (2013). Multisite phosphorylation networks as signal processors for Cdk1. Nat. Struct. Mol. Biol. 20, 1415-1424. doi: 10.1038/nsmb.2706

Kuenzel, E. A., Mulligan, J. A., Sommercorn, J., and Krebs, E. G. (1987). Substrate specificity determinants for casein kinase II as deduced from studies with synthetic peptides. J. Biol. Chem. 269, 9136-9140.

Litchfield, D. W., Arendt, A., Lozeman, F. J., Krebs, E. G., Hargrave, P. A., and Palczewski, K. (1990). Synthetic phosphopeptides are substrates for casein kinase II. FEBS Lett. 261, 117-120. doi: 10.1016/0014-5793(90)80650-8

Marin, O., Meggio, F., Marchiori, F., Borin, G., and Pinna, L. A. (1986). Site specificity of casein kinase-2 (TS) from rat liver cytosol. A study with model peptide substrates. Eur. J. Biochem. 160, 239-244. doi: 10.1111/j.1432-1033.1986.tb09962.x

McConn, M., and Browse, J. (1996). The critical requirement for linolenic acid is pollen development, not photosynthesis, in an Arabidopsis mutant. Plant Cell 8 , 403-416. doi: 10.1105/tpc.8.3.403
Nguyen, H. M., Cuiné, S., Beyly-Adriano, A., Légeret, B., Billon, E., Auroy, P., et al. (2013). The green microalga Chlamydomonas reinhardtii has a single $\omega-3$ fatty acid desaturase that localizes to the chloroplast and impacts both plastidic and extraplastidic membrane lipids. Plant Physiol. 163, 914-928. doi: 10.1104/pp.113.223941

Palczewski, K., Kumasaka, T., Hori, T., Behnke, C. A., Motoshima, H., Fox, B. A., et al. (2000). Crystal structure of rhodopsin: A G protein-coupled receptor. Science 289, 739-745. doi: 10.1126/science.289.5480.739

Pflaster, E. L., Schwabe, M. J., Becker, J., Wilkinson, M. S., Parmer, A., Clemente,T. E., et al. (2014). A high-throughput fatty acid profiling screen reveals novel variations in fatty acid biosynthesis in Chlamydomonas reinhardtii and related algae. Eukaryot. Cell. 11, 1431-1438. doi: 10.1128/EC.00128-14

Routaboul, J. M., Skidmore, C., Wallis, J. G., and Browse, J. (2012). Arabidopsis mutants reveal that short-and long-term thermotolerance have different requirements for trienoic fatty acids. J. Exp. Bot. 63, 1435-1443. doi: 10.1093/jxb/err381

Sasser, M. (1990). Identification of bacteria by gas chromatography of cellular fatty acids. USFCC News. lett. 20, 1-6.

Shanklin, J. (1994). Eight histidine residues are catalytically essential in a membraneassociated iron enzyme, stearoyl-CoA desaturase, and are conserved in alkane hydroxylase and xylene monooxygenase 1. Biochemistry 33, 12787-12794. doi: 10.1021/bi00209a009

Shanklin, J., and Cahoon, E. B. (1998). Desaturation and related modifications of fatty acids. Annu. Rev. Plant Physiol. Plant Mol. Biol. 49, 611-641. doi: 10.1146/annurev.arplant.49.1.611

Shanklin, J., and Somerville, C. (1991). Stearoyl-acyl-carrier-protein desaturase from higher plants is structurally unrelated to the animal and fungal homologs. Proc. Natl. Acad. Sci. U.S.A. 88, 2510-2514. doi: 10.1073/pnas.88.6.2510

Siaut, M., Cuiné, S., Cagnon, C., Fessler, B., Nguyen, M., Carrier, P., et al. (2011). Oil accumulation in the model green alga Chlamydomonas reinhardtii: Characterization, variability between common laboratory strains and relationship with starch reserves. BMC Biotechnol. 11:7. doi: 10.1186/1472-6750-11-7

Wallis, J. G., and Browse, J. (2002). Mutants of Arabidopsis reveal many roles for membrane lipids. Prog. Lipid Res. 41, 254-278. doi: 10.1016/S01637827(01)00027-3

Wathes, D. C., Abayasekara, D. R. E., and Aitken, R. J. (2007). Polyunsaturated fatty acids in male and female reproduction. Biol. Reprod. 77, 190-201. doi: 10.1095/biolreprod.107.060558

Conflict of Interest Statement: The authors declare that the research was conducted in the absence of any commercial or financial relationships that could be construed as a potential conflict of interest.

Received: 29 November 2014; accepted: 19 January 2015; published online: 05 February 2015.

Citation: Lim J-M, Vikramathithan J, Hwangbo K, Ahn J-W, Park Y-I, Choi D-W and Jeong W-J (2015) Threonine 286 of fatty acid desaturase 7 is essential for $\omega-3$ fatty acid desaturation in the green microalga Chlamydomonas reinhardtii. Front. Microbiol. 6:66. doi: 10.3389/fmicb.2015.00066

This article was submitted to Microbiotechnology, Ecotoxicology and Bioremediation, a section of the journal Frontiers in Microbiology.

Copyright (c) 2015 Lim, Vikramathithan, Hwangbo, Ahn, Park, Choi and Jeong. This is an open-access article distributed under the terms of the Creative Commons Attribution License (CC BY). The use, distribution or reproduction in other forums is permitted, provided the original author(s) or licensor are credited and that the original publication in this journal is cited, in accordance with accepted academic practice. No use, distribution or reproduction is permitted which does not comply with these terms. 